\title{
Collective spontaneous emission in a q-deformed Dicke model
}

\author{
Stefano Mancini ${ }^{\dagger}$, Vladimir I. Man’ko and Paolo Tombesi ${ }^{\dagger}$ \\ † Dipartimento di Matematica e Fisica and Unità INFM, \\ Università di Camerino, I-62032 Camerino, Italy \\ ‡Lebedev Physical Institute, Leninsky Pr. 53, 117924 Moscow, Russia
}

(Received: September 16, 2018)

\begin{abstract}
The q-deformation of a single quantized radiation mode interacting with a collection of two level atoms is introduced, analysing its effects on the cooperative behavior of the system.
\end{abstract}

PACS number(s): 03.65.-w, 42.50.Fx

Typeset using REVTEX 


\section{INTRODUCTION}

Since the fundamental paper by Dicke [1] on collective spontaneous emission, a considerable amount of increasing interest has been devoted to an assembly of $N$ two-level atoms located within a distance much smaller than the radiation wavelength. Such a system has commonly been referred to as the Dicke model, in which the cooperative nature of the spontaneous emission is assumed to result from indirect atom-atom coupling via the field mode only.

Since the erliest numerical investigations [2] the model has been known to present two limit cases when it may be treated as an almost linear system. These are called "strong" and "weak" field regimes and have been extensively studied developing several approximation methods [3].

It was also shown [4 that by considering only a fraction $s$ of the $N$ atoms inverted, it is possible to neglect the losses provide to have $s \ll N$. In such a case, the model becomes exactly solvable, up to date, for $s \leq 8$ [5].

On the other hand, the recent development of quantum groups [6] has motivated great interest in q-deformed algebraic structures, in particular the q-oscillators [7]. In this framework, there have been already examples to treat solvable model, like the Jaynes-Cummings, which permits the application of quantum algebra [8].

Here, we shall consider another physically important model of the interaction of a radiation field (q-deformed) with $N$ two-level atoms placed in a lossless single mode cavity, when only a part $s$ of the $N$ atoms radiates spontaneously in the presence of $N-s$ unexcited atoms, and we shall show how the deformation affects the collective phenomena.

In physical terms, this generalization allows us to introduce an additional parameter $q$ into the Dicke model, which shows, therefore, an intensity dependent coupling resembling that one of the Jaynes-Cummings [9], where only one atom is considered.

The physical nature of the additional parameter $q$ might be treated as q-nonlinearity characteristics of the q-oscillator vibrations, for which the frequency of vibrations specifically 
depends on the amplitude of the vibrations [10]. In this case, the nonlinearity of the field vibrations results the intensity-dependent coupling with atoms in a cavity. On the other hand, the intensity-dependent coupling could arise due to a highly nonlinear response of the atom to the action of the linear vibrating-radiation-field oscillator. The use of q-deformed Dicke model, in this case, corresponds to a phenomenological description of the nonlinear interaction of particular atoms with standard electromagnetic field.

Thus, the aim of our work is to study how the nonlinearity described by the q-oscillators influences the known properties of spontaneous emission of atoms in a cavity in the framework of the Dicke model.

\section{THE MODEL}

The Hamiltonian of the system we wish to start with, in rotating wave approximation, can be written as [1] $(\hbar=1)$

$$
\begin{aligned}
H & =H_{0}+V \\
H_{0} & =\omega_{f} a^{\dagger} a+\omega \sum_{j=1}^{N} S_{j}^{(3)} \\
V & =g \sum_{j=1}^{N}\left[a^{\dagger} S_{j}^{-}+a S_{j}^{+}\right]=g\left[a^{\dagger} S^{-}+a S^{+}\right],
\end{aligned}
$$

where $a^{\dagger}(a)$ is the photon creation (annihiliation) operator and $S_{j}^{-}, S_{j}^{+}$, and $S_{j}^{(3)}$ are the pseudo-spin lowering, rising and inversion operators of the $j$ th atom, respectively; $\omega_{f}$ denotes the frequency of the field mode while $\omega$ is the atomic transition frequency. In what follows we assume exact resonance and choose the scale in such a way that $\omega_{f}=\omega=1$. Furthermore, within the small-sample approximation, the coupling coefficient $g$ is the same for all the atoms. The basis vectors of the model read

$$
|s, m\rangle=|s-m\rangle_{a} \otimes|m\rangle_{f},
$$

where $|m\rangle_{f}$ denotes the Fock state of the field, while $|s-m\rangle_{a}$ is the normalized symmetric Dicke state of the atomic subsystem with $s-m$ atoms excited [11]. The initial condition will 
be $|s, 0\rangle$ when $s$ atoms are initially excited and no photon is present (spontaneous emission).

In the cases, $s=1$ and $s=2$, the problem is characterized by equidistant eigenvalues spectra [12], while the latter becomes already unequidistant for $s=3$, and the eigenvalues are incommensurate quantities $[13]$. This unequidistance leads to the phenomenon of collective collapses and revivals of the system oscillations [11].

Let us now introduce the deformation of the field as [10]

$$
\begin{aligned}
V_{q} & =g\left[V_{q}^{+}+V_{q}^{-}\right] ; \\
V_{q}^{+} & =a_{q} S^{+}=a f(n) S^{+} ; \\
f(n) & =f\left(a^{\dagger} a\right)=\sqrt{\frac{\sinh (n \log q)}{n \sinh (\log q)}},
\end{aligned}
$$

with $V_{q}^{-}$the hermitian conjugate of $V_{q}^{+}$; then the nonvanishing matrix elements of these operators are

$$
\begin{aligned}
\left\langle s, m+1\left|V_{q}^{-}\right| s, m\right\rangle & =f(m+1) \sqrt{(m+1)(s-m)(N-s+m+1)} \\
& =\left\langle s, m\left|V_{q}^{+}\right| s, m+1\right\rangle .
\end{aligned}
$$

\section{THE TIME EVOLUTION}

In what follows, for simplicity, we restrict our analysis to the lower three values of $s$. Let us first consider the case of $s=1$, then the system ket state can be written as

$$
|\Phi(t)\rangle=C_{0}(t)|1,1\rangle+C_{1}(t)|1,0\rangle
$$

with the initial condition $C_{0}(0)=0 ; C_{1}(0)=1$. With this choice, the time-dependent Schrödinger equation (in the interaction picture) leads to the coupled equations

$$
\begin{aligned}
& i \dot{C}_{0}=g f(1) \sqrt{N} C_{1} ; \\
& i \dot{C}_{1}=g f(1) \sqrt{N} C_{0} .
\end{aligned}
$$

Since $f(1)=1, \forall q$, the field deformation does not affect the oscillatory solutions. In the case of $s=2$, we have 


$$
|\Phi(t)\rangle=C_{0}(t)|2,2\rangle+C_{1}(t)|2,1\rangle+C_{2}(t)|2,0\rangle
$$

with equations

$$
\begin{aligned}
& i \dot{C}_{0}=g f(2) \sqrt{2 N} C_{1} ; \\
& i \dot{C}_{1}=g f(2) \sqrt{2 N} C_{0}+g f(1) \sqrt{2(N-1)} C_{2} ; \\
& i \dot{C}_{2}=g f(1) \sqrt{2(N-1)} C_{1},
\end{aligned}
$$

whose solutions are

$$
\begin{aligned}
& C_{0}(t)=\frac{2 g f(2) \sqrt{N(N-1)}}{\Omega^{2}}[\cos (\Omega t)-1] ; \\
& C_{1}(t)=\frac{-i \sqrt{2(N-1)}}{\Omega} \sin (\Omega t) ; \\
& C_{2}(t)=\frac{2 g(N-1)}{\Omega^{2}}[\cos (\Omega t)-1]+1,
\end{aligned}
$$

with

$$
\Omega=\sqrt{2 f^{2}(2) N+2(N-1)}
$$

Now, the expectation value of the collective operator $S^{(3)}=\sum S_{j}^{(3)}$, in the eigenstate $|s, m\rangle$ is

$$
\left\langle s, m\left|S^{(3)}\right| s, m\right\rangle=-\frac{N}{2}+s-m
$$

and represents the inversion of the atomic energy for the whole system of $N$ atoms; but in turn we may use the operator $S^{z}=S^{(3)}+(N / 2)-(s / 2)$ obtaining

$$
\left\langle s, m\left|S^{z}\right| s, m\right\rangle=\frac{s}{2}-m
$$

which represents the inversion of the atomic energy for the group of $s$ atoms [1]. Its time evolution can be written as

$$
E_{\mathrm{at}}^{s}(t)=\left\langle\Phi(t)\left|S^{z}\right| \Phi(t)\right\rangle,
$$

and for the case of $s=2$ we get 


$$
E_{\mathrm{at}}^{s=2}(t)=C_{2}^{2}(t)-C_{0}^{2}(t)
$$

It becomes clear from Eq. (15) that the q-nonlinear deformation changes (reduces) the period of oscillations of the quantity (19) making faster the beating of the energy between the atoms and the radiation field in the cavity. Furthermore, the amplitude of these oscillations is reduced towards the upper values as can be seen in Fig. 1; this means that, due to the deformation, there do not exist times at which all initially excited atoms release the photons. In the limit of extremely high deformation, the period of oscillations tends to zero and the value of (19) tends to remain constant (equal to $+s / 2$ ), which implies that for strong nonlinearity the beating phenomenon disappears.

For $s=3$, the analogous of Eq. (8) takes the form

$$
|\Phi(t)\rangle=C_{0}(t)|3,3\rangle+C_{1}(t)|3,2\rangle+C_{2}(t)|3,1\rangle+C_{3}(t)|3,0\rangle,
$$

and the equations for the coefficients become

$$
\begin{aligned}
& i \dot{C}_{0}=g f(3) \sqrt{3 N} C_{1} ; \\
& i \dot{C}_{1}=g f(3) \sqrt{3 N} C_{0}+2 g f(2) \sqrt{N-1} C_{2} ; \\
& i \dot{C}_{2}=2 g f(2) \sqrt{N-1} C_{1}+g \sqrt{3(N-2)} C_{3} ; \\
& i \dot{C}_{3}=g \sqrt{3(N-2)} C_{2} .
\end{aligned}
$$

Their solutions can be written as

$$
\begin{aligned}
C_{0}(t) & =\frac{-2 i f(2) \sqrt{N-1}}{3 g f(3) \sqrt{N(N-2)}} \frac{\Omega_{+}^{2} \Omega_{-}^{2}}{\Omega_{+}^{2}-\Omega_{-}^{2}}\left[\frac{\sin \left(\Omega_{+} t\right)}{\Omega_{+}}-\frac{\sin \left(\Omega_{-} t\right)}{\Omega_{-}}\right] ; \\
C_{1}(t) & =\frac{2 f(2) \sqrt{N-1}}{3 N g^{2} f^{2}(3) \sqrt{3(N-2)}} \frac{\Omega_{+}^{2} \Omega_{-}^{2}}{\Omega_{+}^{2}-\Omega_{-}^{2}}\left[\cos \left(\Omega_{+} t\right)-\cos \left(\Omega_{-} t\right)\right] ; \\
C_{2}(t) & =\frac{-i\left(\Omega_{+}^{2}-3 N g^{2} f^{2}(3)\right) \Omega_{+} \Omega_{-}^{2}}{3 N g^{3} f^{2}(3) \sqrt{3(N-2)}\left(\Omega_{+}^{2}-\Omega_{-}^{2}\right)} \sin \left(\Omega_{+} t\right) \\
& +\frac{i\left(\Omega_{-}^{2}-3 N g^{2} f^{2}(3)\right) \Omega_{+}^{2} \Omega_{-}}{3 N g^{3} f^{2}(3) \sqrt{3(N-2)}\left(\Omega_{+}^{2}-\Omega_{-}^{2}\right)} \sin \left(\Omega_{-} t\right) ; \\
C_{3}(t)= & \frac{\left(\Omega_{+}^{2}-3 N g^{2} f^{2}(3)\right) \Omega_{-}^{2}}{3 N g^{2} f^{2}(3)\left(\Omega_{+}^{2}-\Omega_{-}^{2}\right)} \cos \left(\Omega_{+} t\right)
\end{aligned}
$$




$$
-\frac{\left(\Omega_{-}^{2}-3 N g^{2} f^{2}(3)\right) \Omega_{+}^{2}}{3 N g^{3} f^{2}(3)\left(\Omega_{+}^{2}-\Omega_{-}^{2}\right)} \cos \left(\Omega_{-} t\right)
$$

where

$$
\begin{aligned}
\Omega_{ \pm} & =\frac{g}{\sqrt{2}}\left\{\left(3 f^{2}(3)+4 f^{2}(2)+3\right) N-4 f^{2}(2)-6\right. \\
& \pm\left[\left(9 f^{4}(3)+24 f^{2}(2) f^{2}(3)-18 f^{2}(3)+16 f^{4}(2)+24 f^{2}(2)+9\right) N^{2}\right. \\
& +\left(36 f^{3}(3)-32 f^{4}(2)-24 f^{2}(2) f^{2}(3)-72 f^{2}(2)-36\right) N \\
& \left.\left.+\left(16 f^{4}(2)+48 f^{2}(2)+36\right)\right]^{1 / 2}\right\}^{1 / 2}
\end{aligned}
$$

In this case, the inversion of the atomic energy of Eq. (19) is given by

$$
E_{\mathrm{at}}^{s=3}(t)=\frac{3}{2}-3\left|C_{0}(t)\right|^{2}-2 C_{1}^{2}(t)-\left|C_{2}(t)\right|^{2},
$$

and its behavior is shown in Fig. 2, for various values of the deformation parameter. Substantially, the system oscillates with two incommensurate frequencies, then the phenomenon

of quantum beats leads to modulated oscillations. However, collapses and revivals occur with different period if one introduces the field deformation, and they tend to disappear as soon as one increases the strength of the deformation.

We have used a relatively small value of $N$ in the figures for graphic convenience. It is worth noting that by increasing the value of $N$ only the time scale of the described effects change.

\section{CONCLUSIONS}

In conclusion, we have shown that the introduction of an algeabric q-deformation in the radiation field interacting with a collection of two-level atoms may lead to interesting effects such as the inhibition of the collective spontaneous emission. Really, a large deformation drastically enhances the probability of induced emission/absorption, hence already with very few photons the atoms become saturated and the inversion (of the group of $s$ atoms) 
approaches $s / 2$; from this point of view spontaneous emission is not suppressed, but the probability of reabsorption of spontaneously emitted photons is strongly enhanced.

The analysis has been performed by considering the radiation field initially in a vacuum state, but one could study other cases as well.

Of course the results of this (oversimplifed) model are valid whenever the cavity is engineered to substain exactly only one radiation mode [14], otherwise the field deformation could lead to the decay over other modes.

It is worth noting that in our work the character of the nonlinearity of the deformed Dicke model is described by the specific function $f(n)$ in Eq. (3). Other types of possible nonlinearities were discussed in the framework of nonlinear-oscillator models, for which qoscillators were replaced by f-oscillators with the same structure of annihilation operator but with a different function $f(n)[15]$. As one can see from the solutions to the Schrödinger equation given by (6)-(7), (12)-(14), and (25)-(28), the character of the used nonlinearity does not influence the possibility to solve exactly the Schrödinger equation for the deformed Dicke model, at least for $s=1,2,3$.

Thus, our formulas are valid not only for q-oscillators but for generic f-oscillators of the radiation field interacting with two-level atoms in the cavity (in the framework of Dicke model) as well. For any nonlinearity, for which the function $f(n)$ takes very large values, the quantum beats will be suppressed as it takes place for the case of the discussed q-oscillator model. On the other hand, the concrete choice of the nonlinearity coded in the function $f(n)$ may demonstrate essentially different behavior of the collapses and revivals from the ones given by the standard and q-deformed Dicke models.

\section{ACKNOWLEDGEMENTS}

We acknowledge the financial support of the Istituto Nazionale di Fisica Nucleare. 


\section{REFERENCES}

[1] R. Dicke, Phys. Rev. 93, 99 (1954).

[2] M. Tavis and F. W. Cummings, Phys. Rev. 170, 379 (1968); D. F. Walls and R. Barakat, Phys. Rev. A 1, 446 (1970).

[3] See, e.g., R. Bonifacio and G. Preparata, Phys. Rev. A 2, 336 (1970); M. Kozierowski, S. M. Chumakov, and A. A. Mamedov, Physica A 180, 435 (1992); G. Drobny and I. Jex, Opt. Comm. 102, 141 (1993); S. M. Chumakov, A. B. Klimov and J. J. SanchezMondragon, Phys. Rev. A 49, 4972 (1994); P. L. Knight and B. W. Shore, Phys. Rev. A 48, 642 (1993).

[4] S. M. Barnett and P. L. Knight, Opt. Acta 31, 435 (1984); M. Kozierowski, A. A. Mamedov, and S. M. Chumakov, Phys. Rev. A 42, 1762 (1990).

[5] M. Kozierowski and S. M. Chumakov, Phys. Rev. A 52, 4194 (1995).

[6] See, e.g., V. G. Drinfeld, in: Proceedings of the International Congress of Mathematicians, Berkeley (1986), Vol. 1, p. 798; M. Jimbo, Lett. Math. Phys. 11, 247 (1986).

[7] L. C. Biedenharn, J. Phys. A 22, L873 (1986); A. J. Macfarlane, J. Phys. A 22, 4581 (1986); M. Chaichian and P. Kulish, Phys. Lett. B 234, 72 (1990).

[8] M. Chaichian, D. Ellinas, and P. Kulish, Phys. Rev. Lett. 65, 980 (1990).

[9] B. Buck and C. V. Sukumar, Phys. Lett. A 81, 132 (1981); J. Phys. A 17, 885 (1984); V. Buzek, Phys. Rev. A 39, 3196 (1989).

[10] V. I. Man'ko, G. Marmo, S. Solimeno, and F. Zaccaria, Int. J. Mod. Phys. A 8, 3577 (1993); V. I. Man'ko, G. Marmo, and F. Zaccaria, Phys. Lett. A 197, 95 (1995).

[11] M. Kozierowski, S. M. Chumakov, J. Swiatlowski, and A. A. Mamedov, Phys. Rev. A 46, 7220 (1992).

[12] F. W. Cummings and A. Dorri, Phys. Rev. A 28, 2282 (1983); J. Seke, Phys. Rev. A 
33, 739 (1986); M. Kozierowski and S. M. Chumakov, J. Russ. Laser Research (Plenum Press) 15, 118 (1994).

[13] I. R. Senitzky, Phys. Rev. A 3, 421 (1971).

[14] see e.g., F. de Martini, G. Innocenti, G. R. Jacobovitz and P. Mataloni, Phys. Rev. Lett. 59, 2955 (1987).

[15] V. I. Man'ko, G. Marmo, E. C. G. Sudarshan, and F. Zaccaria, in Proceedings of the Fourth Wigner Symposium (Guadalahara, Mexico, July 1995), eds. N. M. Atakishiyev, T. H. Seligman, and K.-B. Wolf (World Scientific, Singapore, 1996), p. 421; Physica Scripta, 55, 528 (1997). 


\section{FIGURE CAPTIONS}

Fig. 1 Time evolution of the atomic inversion for $N=6$ and $s=2$. The deformation parameter takes the values: a) $q=1$; b) $q=5$; c) $q=20$.

Fig. 2 Time evolution of the atomic inversion for $N=6$ and $s=3$. The deformation parameter takes the values: a) $q=1$; b) $q=2$; c) $q=4$. 